\title{
CORRECTION
}

\section{Correction to: Effective behavior of cooperative and nonidentical molecular motors}

\author{
Joseph J. Klobusicky, John Fricks ${ }^{2}$ and Peter R. Kramer ${ }^{3}$
}

\section{Correction to:}

\section{Effective behavior of cooperative and nonidentical molecular motors https://doi.org/10.1007/s40687-020-00230-7}

In the original article the drift terms in Eqs. (2) and (4) and the Hookean spring relation in the paragraph following Eq. (4) have sign errors. These errors are only typos and do not propagate to other equations in the manuscript. The corrected equations and paragraph follow:

$$
\begin{aligned}
& \gamma d Z(t)=\sum_{j=1}^{N} F^{(j)}\left(X^{(i)}(t)-Z(t)\right) d t-F_{T} d t+\sqrt{2 k_{B} T_{\gamma}} d W_{z}(t) . \\
& \mu_{d}^{(i)}(x, z)=-F^{(i)}(x-z) / \gamma_{m}^{(i)} .
\end{aligned}
$$

If $Q^{(i)}(t)=1$, equation (1) describes an attached motor with position $X^{(i)}(t)$. The restorative force in (3) results from the stretching of the coiled-coil tether that connects the motor head and tail attached to the cargo. While a nonlinear force model for the tether would seem to be most appropriate $[27,59,65]$, we could not find a clear consensus on its precise form. To reduce technical complications in the formulas and to keep focus on the overall structure of the mathematical course-graining, in the present work, similarly to Miles et al. [31], we model the force for this tether for motor $i$ by a simple Hookean spring relation $F^{(i)}(y)=\kappa^{(i)} y$, where $y$ is the longitudinal displacement from the cargo to the motor, and $\kappa^{(i)}$ is the effective spring constant for the tether to the $i$ th motor. The value we have cited from Furuta, Furuta et al. [32] is measured from motors attached to a DNA scaffold, but we expect the stiffness to mostly reflect the properties of the motor tether [30]. The value is roughly consistent with the $\kappa \approx 0.3 \mathrm{pN} / \mathrm{nm}$ values found in other experiments $[66,54,65]$. Surely a better model for the tether would have it be slack under compression from its rest length $[54,67,56,68,32]$. While we do not investigate 
the consequences of nonlinear tether force models here in detail, our framework can be generalized to include them, as discussed in Appendix A.

The original article can be found online at https://doi.org/10.1007/s40687-020-00230-7.

Published online: 9 February 2021

\section{Publisher's Note}

Springer Nature remains neutral with regard to jurisdictional claims in published maps and institutional affiliations. 